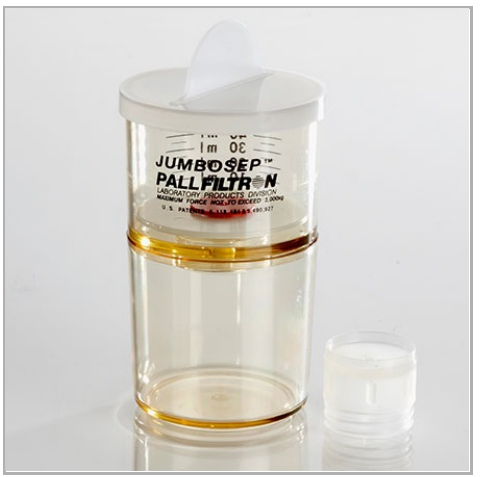

SEP 19, 2020

open Әaccess

DOI:

dx.doi.org/10.17504/protocol s.io. beqnjdve

Protocol Citation: Joshua A Welsh, Julia Kepley, Bryce Killingsworth, Tim Traynor, Jennifer Jones 2020.

Ultrafiltration and purification of conditioned media (Pall Jumbsosep and Izon qEV-10) . protocols.io

https://dx.doi.org/10.17504/p rotocols. io. beqnjdve

License: This is an open access protocol distributed under the terms of the Creative Commons Attribution License, which permits unrestricted use, distribution, and reproduction in any medium, provided the original author and source are credited

Protocol status: Working We use this protocol and it's working

Created: Apr 07, 2020

Last Modified: Sep 23, 2020

PROTOCOL integer ID: 35310

\section{(3) Ultrafiltration and purification of conditioned media (Pall Jumbsosep and Izon qEV-10)}

\author{
Joshua A Welsh ${ }^{1}$, Julia Kepley ${ }^{1}$, Bryce Killingsworth ${ }^{1}$, \\ Tim Traynor $^{1}$, Jennifer Jones ${ }^{1}$ \\ ${ }^{1}$ Translational Nanobiology Section, Laboratory of Pathology, Center for \\ Cancer Research, National Cancer Institute, National Institutes of Health
}

Translational Nanobiology Section

Jennifer Jones

\section{DISCLAIMER}

This protocol summarizes key steps for a specific type of method, which is one of a collection of methods and assays used for EV analysis in the $\mathrm{NCl}$ Translational Nanobiology Section at the time of submission of this protocol. Appropriate use of this protocol requires careful, cohesive integration with other methods for EV production, isolation, and characterization.

\section{ABSTRACT}

Protocol for ultrafiltration of cell culture conditioned media using Pall Jumbosep products and purification of EVs from conditioned media concentrate using Izon qEV10 size exclusions columns.

\section{GUIDELINES}

- All handling of conditioned media for isolation and purification of EVs should be done in sterile conditions

- Each additional $60 \mathrm{~mL}$ of conditioned media that is filtered through the Jumbsosep device insert increases the time required to concentrate subsequently added media to the device

\section{MATERIALS}

MATERIALS

$880.5 \mathrm{M} \mathrm{NaOH}$ Contributed by users

88 DPBS, no calcium, no magnesium Thermo Fisher Catalog \#14190136

Ethanol Contributed by users

For concentrating conditioned media: 
- An unopened sterile $500 \mathrm{~mL}$ bottle of Gibco DPBS

- $70 \mathrm{~mL}$ of HPLC or other pure water

- $30 \mathrm{~mL}$ of Ethanol

- Pall Jumbosep filtrate receiver, sample reservoir, and 100k membrane insert

- 3 Eppendorf $5 \mathrm{~mL}$ low protein binding tubes

- Centrifuge inserts to hold Jumbosep devices

\section{Jumbosep ${ }^{\mathrm{TM}}$ Centrifugal Devices}

Starter Kits with Omega PES Membrane

\begin{tabular}{|c|c|c|}
\hline Product No. & Description & Packaging \\
\hline FD000K65 & Generic starter kit, (no membrane or inserts) & $4 / \mathrm{pkg}$ \\
\hline FD003K65 & 3K starter kit, gray & $4 / \mathrm{pkg}$ \\
\hline FD010K65 & $10 \mathrm{~K}$ starter kit, blue & $4 / \mathrm{pkg}$ \\
\hline FD030K65 & $30 \mathrm{~K}$ starter kit, red & $4 / \mathrm{pkg}$ \\
\hline FD100K65 & $100 \mathrm{~K}$ starter kit, clear & $4 / \mathrm{pkg}$ \\
\hline FD300K65 & $300 \mathrm{~K}$ starter kit, orange & $4 / \mathrm{pkg}$ \\
\hline
\end{tabular}

\section{Jumbosep Device Omega PES Membrane Inserts}

\begin{tabular}{|c|c|c|}
\hline Product No. & Description & Packaging \\
\hline $0 \mathrm{D003C65}$ & $3 \mathrm{~K}$ membrane insert, gray & $12 / p k g$ \\
\hline 0D010C65 & $10 \mathrm{~K}$ membrane insert, blue & $12 / \mathrm{pkg}$ \\
\hline 0D030C65 & $30 \mathrm{~K}$ membrane insert, red & $12 / p k g$ \\
\hline 0D100C65 & $100 \mathrm{~K}$ membrane insert, clear & $12 / \mathrm{pkg}$ \\
\hline 0D300C65 & $300 \mathrm{~K}$ membrane insert, orange & $12 / \mathrm{pkg}$ \\
\hline
\end{tabular}

\section{Accessory Products}

\begin{tabular}{|c|c|c|}
\hline Product No. & Description & Packaging \\
\hline FD001X65 & Filtrate receiver and cap & $12 / \mathrm{pkg}$ \\
\hline FD002X65 & Sample reservoir and cap & $12 / p k g$ \\
\hline FD003X65 & Insert release & $24 / \mathrm{pkg}$ \\
\hline
\end{tabular}

Table of available Pall Jumbsosep products (Jan 2020)

For concentrate purificiation with qEV column:

- Izon qEV-10 Column (70 nm pore size)

- New $500 \mathrm{~mL}$ of bottle Gibco sterile DPBS

- 14 x $5 \mathrm{~mL}$ LoBind Eppendorf tubes

- $20 \mathrm{~mL}$ of filterd $20 \%$ ethanol 
- $\sim 5 \mathrm{~mL}$ of filtered $0.5 \mathrm{M} \mathrm{NaOH}$

\section{Preparing the Pall Jumbosep device}

1 Make up 70\% ethanol with HPLC or other pure water

2 Filter at least $20 \mathrm{~mL}$ of the $70 \%$ ethanol with a $0.02 \mu \mathrm{m}$ syringe-driven filter, or other small pore size filter

3 Obtain a Jumbosep sample reservoir, a Jumbsosep filtrate receiver, and a Jumbosep insert

4 Snap the reservoir onto the receiver

5 Without contacting the inside of the reservoir or the insert, place the insert into the reservoir

6 Use the blunt end of an aspirating tip to firmly push the insert into place

7 Fill the reservoir with at least $10 \mathrm{~mL}$ of the prepared filtered $70 \%$ ethanol

8 Place the reservoir cap onto the reservoir 
9 Swirl the device around to maximize ethanol contact and let sit for a 5 minutes

10 Spin the device (with an appropriate swinging bucket rotor and adaptor insert, along with another balancing device, as needed) at 2500 RCF for 5 minutes to concentrate ethanol down to the device's dead volume of $5 \mathrm{~mL}$

11 To separate the assembled device, remove the reservoir cap, clasp hands behind the device, place both thumbs on the lip of the sample reservoir, and press upward and backward.

12 Use a tissue to blot any fluid which may be present on rim of reservoir and receiver

13 Flush the reservoir with $\sim 10 \mathrm{~mL}$ of sterile PBS and aspirate all volume from the receiver

14 Assemble the device, add $60 \mathrm{~mL}$ of sterile PBS to the reservoir and spin at $3000 \mathrm{RCF}$ for 5 minutes

15 Aspirate all volume from the receiver, and the device is ready to have conditioned media loaded onto it

\section{Cell culture media clearing}


16 Cell culture media is ready to be harvested from cells when they are $50 \%$ to $80 \%$ confluent, typically 48 hours after cells have been seeded into phenol-red-free, bovine EV-depleted complete media

17 Using a $50 \mathrm{~mL}$ serological pipet tip, transfer the complete volume of media in each flask of cells to a sterile $50 \mathrm{~mL}$ conical tube

18 Spin the tubes at 500 RCF for 10 minutes

19 Transfer the supernatants from the $50 \mathrm{~mL}$ conical tubes to a new set $50 \mathrm{~mL}$ conical tubes, leaving behind a small but visible pellet of cells and cell debris

20 Spin the tubes at 2500 RCF for 10 minutes

21 Transfer the supernatants from the $50 \mathrm{~mL}$ conical tubes (leaving behind 1 to $2 \mathrm{~mL}$ of fluid at the bottom of the tubes) to a sterile media storage bottle for temporary storage during the subsequent ultrafiltration process

\section{Concentrating cleared conditioned media}

22 Wipe down with $70 \%$ ethanol two $750 \mathrm{~mL}$ centrifuge bucket inserts and the insides of the $750 \mathrm{~mL}$ centrifuge buckets

23 Load conditioned media into the reservoir until the volume reaches the $60 \mathrm{~mL}$ mark 
24 Spin the device at 3000 RCF for $\sim 20$ minutes checking to ensure only the $\sim 5 \mathrm{~mL}$ dead volume of fluid remains in the sample reservoir after the spin

25 Disasemble the device, aspirate the filtrate, and reasemble the device before addition of conditioned media

26 Add conditioned media to the sample reservoir until it reaches the $60 \mathrm{~mL}$ mark

27 If you are trying to balance two devices but have less than $60 \mathrm{~mL}$ of media for each device, PBS can be added to one of the devices to ensure the volumes are equal

28 Spin at $3000 \mathrm{RCF}$ for 30 minutes at room temperature or until only $\sim 5 \mathrm{~mL}$ of fluid remains in the sample reservoir

29 Continue the process of adding additional cleared conditioned media until all media has been transferred to the sample reservoir, increasing the centrifuge time as necessary (typically 5 to 10 minutes more per additional spin)

30 If the last volume of media remaining to be filtered is not $60 \mathrm{~mL}$, PBS can be added to the reservoir to fill it to the $60 \mathrm{~mL}$ marker

31 After the last spin with added cleared media, fill the sample reservoir with $60 \mathrm{~mL}$ of PBS, ensuring resuspension of any material adhered to the bottom of the reservoir and insert, and complete the final $3000 \mathrm{RCF}$ spin at RT, so that there is a $\sim 5 \mathrm{~mL}$ dead volume 
This is a wash step to remove any soluble protein $<100 \mathrm{kDa}$ that may be remaining in the 5 $\mathrm{mL}$ dead volume.

32 Being careful not to spill concentrate or create excessive bubbles, use a P1000 micropipette to thoroughly resuspend the concentrate which is sitting on top of the insert in the sample reservoir

33 Transfer the $\sim 5 \mathrm{~mL}$ from the top of the sample reservoir to Eppendorf Low-protein binding $5.0 \mathrm{~mL}$ tubes, placing a maximum volume of $4 \mathrm{~mL}$ in each tube to prevent overflow

34 Label and store the conditioned media concentrate at $4{ }^{\circ} \mathrm{C}$ overnight, do not store for more than a short period of time without purifying the concentrate

\section{Size exclusion chromatography of conditioned media conce.}

35 Remove a new qEV-10 column from its $4^{\circ} \mathrm{C}$ storage location at the start of work day to allow to warm to room temperature

36 Use a ring stand and metal clamp to position the column vertically

37 Rinse $5 \mathrm{~mL}$ of PBS from a new, unopened, room temperature bottle of PBS through the loading chamber into a waste container

38 Unscrew the top cap from the column 
39 Fill the column with PBS until it fills the column

40 Press the loading chamber into the top of the column, ensuring a tight seal between the two units

$41 \quad$ Fill the loading chamber to the top with PBS

42 Unscrew the bottom cap from the column, PBS should flow through the column and into a waste container

43 Once around half the PBS volume in the loading chamber has flushed through the column, fill it back up to the top with PBS

44 While the column is being flushed, label 14, $5 \mathrm{~mL}$ low protein-binding Eppendorf tubes with the a fraction number ( 1 through 14) and date. A marker can be used to highlight the $5.0 \mathrm{~mL}$ volume graduation on the tube

45 As soon as all of the loaded PBS has moved through the loading chamber, position the "Fraction 1" $5.0 \mathrm{~mL}$ tube under the colum. Gently vortex and invert the concentrated media and add it to the loading chamber $1 \mathrm{~mL}$ at a time, ensuring to pipette the media directly onto the reservoir filter and not the walls of the chamber. The time elapsed from the last drop of PBS eluting to loading the cell culture media concentrate onto the column should be as short as possible.

46 As soon as all of the loaded concentrated media has passed through the loading chamber, the loading reservoir can be filled to the top with PBS 
47 Continue collecting fractions in $5.0 \mathrm{~mL}$ increments until all fractions have been collected, refilling the loading chamber to the top with PBS when the PBS level falls by about half

48 While PBS continues to flush through column, into a waste container, collected fraction tube lids can be closed and set aside

49 Once the entire volume of PBS in the loading chamber has run through, add $5 \mathrm{~mL}$ of filtered $0.5 \mathrm{M}$ $\mathrm{NaOH}$ to the chamber and allow it to pass through the column. The $\mathrm{NaOH}$ can be added by pipetting along the walls of the reservoir to wash away any contaminants

50 Fill the buffer reservoir to the top with PBS and allow it all to pass through the column except for the last $\sim 1 \mathrm{~mL}$ to prevent drying the column

51 Refill the buffer reservoir to the top with PBS

52 After the majority of the buffer has eluted use a pH test strip to ensure the eluting buffer is neutral

53 Fill the column up with $5 \mathrm{~mL}$ of filtered $20 \% \mathrm{EtOH}$ and allow it to flush through column

54 Place the bottom cap back on the column

55 Pipette filtered $20 \% \mathrm{EtOH}$ into the top of the column until it is filled to the top with EtOH. 
56 Place the top cap back on the column

57 Allow the loading chamber to dry in the hood overnight

58 Return all column components to the box they came from and lable the box with date used, cell line used on, and volume of concentrated media loaded into the column. 